\title{
PERIPARTUM ANAESTHETIC MANAGEMENT OF TWIN GESTATIONAL PREGNANCY IN PATIENT WITH TAKAYASU'S ARTERITIS
}

\author{
Praveen Prasad ${ }^{1}$, Archana Jagadish Pai²
}

${ }^{1}$ Visiting Consultant, Department of Anaesthesiology, Cloudnine Hospital, Malleswaram, Bangalore. ${ }^{2}$ Registrar, Department of Anaesthesiology, Cloudnine Hospital, Malleswaram, Bangalore.

HOW TO CITE THIS ARTICLE: Praveen P, Archana JP. Peripartum anaesthetic management of twin gestational pregnancy in patient with Takayasu's arteritis. J. Evolution Med. Dent. Sci. 2017;6(84):5887-5889, DOI: 10.14260/Jemds/2017/1279

\section{PRESENTATION OF CASE}

Takayasu's aortoarteritis (TA) is a chronic, progressive, idiopathic, occlusive arteritis of the aorta and aortic arch branches. ${ }^{1}$ It is commonly seen in women of reproductive age group. Peripartum management of pregnant patients with TA is challenging and must be approached with careful vigilance due to the high rate of hypertensive complication, multiorgan involvement and foetal complications. These influence the timing, mode of delivery and also the type of anaesthesia administered.

We present a 22-year-old primgravida with twin gestation with known TA with stents in situ, who delivered at our institution by elective LSCS at 34 weeks by spinal anaesthesia.

\section{DIFFERENTIAL DIAGNOSIS}

The following are the differential diagnoses for Takayasu's arteritis,

1. Aortic Coarctation.

2. Giant Cell Arteritis.

3. Buerger Disease.

4. Atherosclerosis.

5. Rheumatoid Arthritis.

6. Behcet Disease.

7. Systemic Lupus Erythematosus.

8. Wegener Granulomatosis.

9. Sarcoidosis.

10. Kawasaki Disease.

\section{CLINICAL DIAGNOSIS}

A 22 yr. old woman $(G 1, P 0)$ married since 3 yrs. was examined by our anaesthetic team at 33 weeks of pregnancy in the PAC clinic. Patient is a known case of TA diagnosed at the age of 16 yrs. At that time, she complained of symptoms of acute breathlessness, chest pain and claudication pain in both lower extremities. CT Angiography revealed $80 \%-90 \%$ stenosis of the infrarenal abdominal aorta, suggestive of aortoarteritis. Patient underwent percutaneous transluminal angioplasty stenting. After nearly 4 years, patient developed recurrence of above symptoms with associated history of palpitations. Repeat CT angiography showed $60 \%-70 \%$ stenosis of the descending thoracic aorta and $25 \%-30 \%$ of left vertebral artery. Re-stenting of the descending aorta was completed successfully and she was continued on antihypertensive and anticoagulation therapy according to the physician's advice.

Financial or Other, Competing Interest: None.

Submission 17-08-2017, Peer Review 07-10-2017,

Acceptance 12-10-2017, Published 19-10-2017.

Corresponding Author:

Dr. Praveen Prasad,

\#47, 17 ${ }^{\text {th }}$ Cross, $11^{\text {th }}$ Main,

Malleshwaram, Bangalore-560055.

E-mail:drsnpp@yahoo.com

DOI: $10.14260 /$ jemds $/ 2017 / 1279$

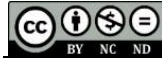

Patient conceived through Assisted Reproductive Technology (ART). Her $8^{\text {th }}$ week ultrasound scan revealed a dichorionic diamniotic twin gestation. Patient was given the option of foetal reduction in view of the added complications of twin gestational pregnancy on her condition. However, patient wanted to continue the same. She was started on alphadopa (metoprolol stopped during pregnancy) and continued on ecosprin.

During consultation at 33 weeks of gestation, patient complained of headache and occasional breathlessness. On examination, blood pressures were found increased to range of 160 - 180 systolic and diastolic of $105-110 \mathrm{mmHg}$, heart rate of 105 beats per min, pulses diminished on lower limbs though waveform by pulse oximetry were normal. Chest examination was clear with no added sounds. Urine for albumin dipstick method was 2+. Cardiology references with an abdominal arterial Doppler done showed aortic stent in situ and normal flow in both the lower limbs. Echocardiography study was normal with an EF of $65 \%$. Her antihypertensives were increased accordingly. Steroid prophylaxis was administered to the patient.

On completion of 34 weeks, she was posted for elective LSCS in view of superimposed preeclampsia and breech presentation of both twins. On the day of surgery, standard fasting guidelines were followed and antacids administered. Prior to the spinal injection, arterial line was secured in the left radial artery using ultrasound guidance, pulse oximetry in upper and lower limbs, 5-electrode ECG and urine output. Her baseline arterial BP was 160/98 mmHg with heart rate of 90 bpm. Pulse oximetry waveform was normal on both limbs. Care was taken to avoid acute flexion of the head while positioning for the spinal injection.

Spinal anaesthesia was administered in the L3-L4 interspinous space using a 27G Quincke needle. CSF free flow confirmed and approximately $1.8 \mathrm{~mL}$ of $0.5 \%$ hyperbaric bupivacaine with $25 \mathrm{mcg}$ of fentanyl was injected in the subarachnoid space. A T4 level of block was achieved. A wedge was placed under the right hip to maintain the left lateral tilt. Two live male babies with birth weight of 1900 grams and 1740 gram were extracted.

Oxytocin infusion was started with 10 units in $500 \mathrm{~mL}$ of ringer lactate solution. Patient received a total of 1.5 litres of fluids throughout the surgery.

Patient remained haemodynamically stable intraop. Blood pressure drop post anaesthesia was within $20 \%$ preanaesthesia BP range and hence vasopressors were not required. She was carefully monitored in the post-operative ICU to avoid hypo/hypertensive complications. For immediate postop analgesia, diclofenac and paracetamol suppositories were placed.

On the postop day 1, her antihypertensives were adjusted according to physician's advice. Adequate round the clock 
analgesia was given. She was discharged from the hospital after 3 days with no untoward incident.

\section{PATHOLOGICAL DISCUSSION}

Macroscopically, in the chronic phase the aorta is thickened secondary to fibrosis of all three vessel layers. The lumen is narrowed in a patchy distribution, often affecting multiple areas. If disease progression is rapid, fibrosis can be inadequate with subsequent aneurysm formation. The intima may be ridged with a "tree bark" appearance, a feature common to many aortitides. Microscopically, the vasculitis may be divided into an acute florid inflammatory phase and a healed fibrotic phase. In the acute phase, a vasa vasoritis is seen in the adventitia. The media is infiltrated by lymphocytes and occasional giant cells with neovascularisation. Mucopolysaccharides, smooth muscle cells and fibroblasts thicken the intima. In the chronic phase, there is fibrosis with destruction of elastic tissue.

\section{DISCUSSION OF MANAGEMENT}

TA, a chronic disease of unknown aetiology, affects the arteries causing narrowing and reduced blood flow to the tissues. Most common incidence is seen in Japan, East and South Asia and India. ${ }^{2}$ Japanese patients were found to be having the "pulseless" disease, whereas many Indian patients were "hypertensive" due to the disease. The location of the disease exhibited variation when investigated by angiography. Indian patients were found to have the disease involvement in the abdominal aorta involving the renal arteries subsequently extending into the thoracic aorta, whereas the Japanese patients had involvement of the aortic arch and its branches. ${ }^{3}$

Pregnancy does not change the natural progression of the disease, but the hypertensive complications like preeclampsia, chronic hypertension, miscarriage and foetal death have been reported in $60 \%-90 \%$ of the cases. IUGR is seen in $20 \%-50 \%$ of the cases.4,5,6,7 In our patient, the disease involved the infrarenal and descending thoracic aorta. Such condition can severely reduce iliac artery blood flow and can restrict uterine perfusion as well as cause progressive foetal growth restriction. ${ }^{4}$ However, in our patient, abdominal arterial Doppler scan suggested adequate flow in iliac arteries with no evidence of uterine hypoperfusion. Umbilical artery Doppler study was also normal. Foetal weight of the twins was corresponding to the gestational age throughout pregnancy with no evidence of IUGR.

Twin gestational pregnancy causes exaggerated physiological changes in mother. The maternal blood volume increases by $500 \mathrm{~mL}$ more than the usual and cardiac output is greater. The supine hypotension syndrome is also more significant with larger uterine size. These factors are to be considered in the over stressed maternal circulation with the preexisting disease.

Anaesthetic management of TA focuses on maintaining haemodynamic stability in patients with compromised regional circulation due to arterial stenosis. Both increase and decrease in systemic vascular resistance can have detrimental effect. Labour pain, pushing during labour and endotracheal intubation cause increase in sympathetic tone which can cause increase in SVR and exaggerate hypertension leading to cardiac failure and cerebral haemorrhage. Decrease in SVR can have negative effect on regional perfusion and causes hypotension induced cerebral, renal, intestinal and uterine ischaemia. ${ }^{4}$

Previous reports in similar cases have used techniques including epidural, spinal, combined spinal-epidural and even general anaesthesia. The ideal choice being regional technique. This helps to monitor cerebral perfusion when patient is awake. In our patient, we preferred use of spinal anaesthesia with $1.8 \mathrm{~mL}$ of $0.5 \%$ hyperbaric bupivacaine with $25 \mathrm{mcg}$ fentanyl. The choice of anaesthesia in our patient was taken with consideration that the lower abdominal vessels were involved in TA and spinal anaesthesia induced vasodilation may improve the circulation in lower extremities. Arterial monitoring is not mandatory for TA patients undergoing surgery due to the risks of pseudoaneurysms ${ }^{4}$ with arterial cannulation. However, to avoid any discrepancy with the NIBP between upper limbs and lower limbs BP in this patient with superimposed preeclampsia, we chose to have the intraarterial cannula present. It was kept for only a short duration in the post-operative period and later removed.

Level of block at T4 level was achieved. Fall in BP more than $20 \%$ of the baseline was considered for treatment with Inj. Ephedrine $6 \mathrm{mg}$. Oxytocin infusion was maintained at approximately 30 - 40 drops per min (10 IU in $500 \mathrm{~mL}$ fluid) and rapid administration avoided to prevent hypotension. Fluid administration was titrated accordingly keeping caution to prevent acute pulmonary oedema. Regardless of the technique or drug used, maintaining adequate arterial perfusion pressure during perioperative period remains the primary goal in these patients.

In conclusion, every pregnant woman with TA should be considered as high-risk pregnancy irrespective of the present disease state. Recurrence of disease symptoms or superimposed complications makes these patients vulnerable to life-threatening risks. Involvement of anaesthesiologists early in the pregnancy helps to optimise patients and formulate a plan of delivery in emergency and elective cases.

\section{FINAL DIAGNOSIS}

Takayasu's arteritis is a chronic disease of the arteries. Pregnancy further complicates the pathology of the disease. Patients with Takayasu's arteritis and pregnancy must be treated as high risk case. Preopertaive evaluation and proper planning is essential for the same.

\section{REFERENCES}

[1] Kerr GS, Hallahan CW, Giordano J, et al. Takayasu arteritis. Ann Intern Med 1994;120(11):919-29.

[2] Hunder GG. Classification and approach to the vasculitides in adults. 2006. Disponível em: http://www.uptodate.com.

[3] Moriwaki R, Noda M, Yajima M, et al. Clinical manifestations of Takayasu arteritis in India and Japannew classification of angiographic findings. Angiology 1997;48(5):369-79.

[4] Ioscovich A, Gislason R, Fadeev A, et al. Peripartum anesthetic management of patients with Takayasu's arteritis: case series and review. International Journal of Obstetric Anesthesia 2008;17(4):358-64.

[5] Sharma BK, Jain S, Vasishta K. Outcome of pregnancy in Takayasu arteritis. Int J Cardiol 2000;75(Suppl 1): S159-S62. 
[6] Matsumura A, Moriwaki R, Numano F. Pregnancy in Takayasu arteritis from the view of internal medicine. Heart Vessels 1992;(Suppl 7):120-4.
[7] Wong VC, Wang RY, Tse TF. Pregnancy and Takayasu's arteritis. Am J Med 1983;75(4):597-601. 